\title{
A methodology for studying physical and dynamical properties of multiple stars. Application to the system of red dwarfs GI 22
}

\author{
J. A. Docobo ${ }^{1}$, V. S. Tamazian ${ }^{1}$, Y. Y. Balega ${ }^{2}$, M. Andrade ${ }^{1}$, D. Schertl ${ }^{3}$, G. Weigelt ${ }^{3}$, P. Campo ${ }^{1}$, and M. Palacios ${ }^{4}$ \\ 1 Observatorio Astronómico Ramón María Aller, Universidade de Santiago de Compostela, Avenida das Ciencias s/n., \\ Santiago de Compostela, Spain \\ e-mail: [oadoco; oatamaz; oandrade; oapedro] @usc.es \\ 2 Special Astrophysical Observatory, N. Arkhyz, Karachai-Cherkasia 369167, Russia \\ e-mail: balega@sao.ru \\ 3 Max-Planck-Institut für Radioastronomie, Auf dem Hügel 69, 53121 Bonn, Germany \\ e-mail: [ds; weigelt]@mpifr-bonn.mpg.de \\ 4 Grupo de Mecánica Espacial, Universidad de Zaragoza, Spain \\ e-mail: mpala@unizar.es
}

Received 31 August 2007 / Accepted 30 October 2007

\section{ABSTRACT}

\begin{abstract}
Aims. The main aim of this study is the elaboration of a methodology for studying physical and dynamical properties of multiple stars and its application to the hierarchical triple system Gl 22. A careful evaluation of the components' masses and a study of the system's overall stability and long-term dynamical evolution were also pursued.

Methods. New NIR speckle interferometric observations with the $6 \mathrm{~m}$ telescope of the Special Astrophysical Observatory (Russia) in the $K^{\prime}$ photometric band have been carried out. We have made use of the method for orbit calculation reported by Docobo (1985).

Results. An original methodology was elaborated and applied to evaluate the most probable elements of the outer orbit. Due to the almost definitive orbit of the inner pair, which just covered a full revolution, the motion of $\mathrm{B}$ relative to $M C_{A}$ has been carefully calculated. The position of $M C_{A}$ was estimated on the basis of differential photometry and empirical mass-luminosity relationships. A weak sinusoidal pattern in the apparent motion of the component B was noticed.

Conclusions. Our methodology was successfully applied to the triple system Gl 22. The newly calculated outer orbit exhibits a moderate eccentricity $(e=0.29)$, which differs from the previously known circular solutions. Both orbits are coplanar and corevolving. This already known suggestion is now based on a much larger set of observational data, including a significant number of speckle measurements. Gl 22 is most likely a dynamically stable system, at least on the time scale of 10 Myr. The sinusoidal pattern in the motion of the B component could be caused by a fourth, unseen, very low-mass object with a mass of $0.015 M_{\odot}\left(16 M_{J}\right)$ on a circular orbit around B with a period of $\sim 15 \mathrm{yr}$ and semimajor axis 0.35 .
\end{abstract}

Key words. astrometry - stars: binaries: visual - stars: individual: Gl 22 - stars: fundamental parameters - stars: late-type - stellar dynamics

\section{Introduction}

Visual companion B, at a distance of 2'. 3 from the nearby bright star Gl 22 (HIP 2552; ADS 440), was discovered by Espin \& Milburn (1926). Using the photographic plates obtained at Leander McCormick Observatory, Alden (1947) reported the primary component A to be an astrometric binary (hereafter components $\mathrm{Aa}$ and $\mathrm{Ab}$ ) itself. On the basis of red/infrared spectra (6300 to $9000 \AA$ ), spectral types M2.0V and M3.0V were assigned to the combined spectrum of the $\mathrm{Aa}+\mathrm{Ab}$ and $\mathrm{B}$ components, respectively (Kirkpatrick et al. 1991). Throughout this paper, pairs $\mathrm{Aa}-\mathrm{Ab}$ (with its mass center $M C_{A}$ ) and $M C_{A}-B$ will be referred to as the inner and the outer orbits, respectively (with the corresponding definition of the inner and outer orbits).

Hershey (1973) published first orbits for the outer $\left(a=4{ }^{\prime \prime} 1\right.$, $P=320 \mathrm{yr}, e=0.05)$ and inner $\left(\alpha=0{ }^{\prime} 125, P=15.95 \mathrm{yr}\right.$, $e=0.0)$ pairs and reached the conclusion that the two orbits are coplanar and the stars are co-revolving. At the same time, he obtained estimates for the total mass of the $\mathrm{Aa}-\mathrm{Ab}$ system $\left(0.53 M_{\odot}\right)$ and the B component $\left(0.12 M_{\odot}\right)$.
Prior to this, on the basis of a photographic plate collection obtained at Sproul Observatory, Heintz (1955) measured an absolute parallax of 0 ' 102 for Gl 22. Furthermore, with a larger set of plates, Heintz (1993) recalculated the orbits of Hershey, but their circular characters remained the same.

Using near-infrared (NIR) speckle interferometry techniques, McCarthy et al. (1991) presented the first two directly resolved observations of the inner pair. The improved inner and outer orbits, both being circular, were obtained by Söderhjelm (1999) and Lampens \& Strigachev (2001), respectively.

With our most recent measurements presented in this paper, the inner pair has now covered a full revolution. They demonstrate very small residuals with regard to our most recently calculated orbit (Docobo \& Tamazian 2006) which is likely to be close to definitive. While the outer pair covered an arc of about $80^{\circ}$, it seemed reasonable to calculate its most probable orbital elements using an original methodology, described below.

Taking into account new orbital elements of both pairs, we studied the system's dynamical stability by the application of several well-known stability criteria. Furthermore, we analysed 


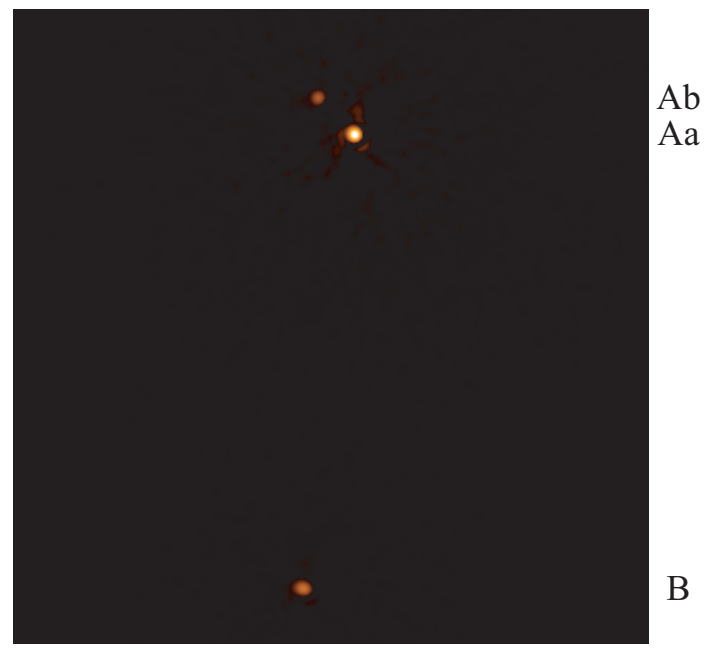

Fig. 1. Diffraction-limited image of Gl 22 in the $K^{\prime}$ band (the components $\mathrm{Aa}, \mathrm{Ab}$ and $\mathrm{B}$ are marked). Field of view 5'.5, north is up, and east is to the left.

Table 1. Astrometry and differential photometry in the $K^{\prime}$ band.

\begin{tabular}{ccccc}
\hline \hline Comp. & Epoch & $\begin{array}{c}\rho \\
(\mathrm{mas})\end{array}$ & $\begin{array}{c}\theta \\
(\mathrm{deg})\end{array}$ & Intens. ratio \\
\hline $\mathrm{Ab} / \mathrm{Aa}$ & 2005.803 & $451 \pm 9$ & $45.5 \pm 1.5$ & $0.143 \pm 0.005$ \\
$\mathrm{~B} /(\mathrm{Aa}+\mathrm{Ab})$ & 2005.803 & $3954 \pm 20$ & $173.7 \pm 0.5$ & $0.235 \pm 0.005$ \\
\hline
\end{tabular}

the long-term evolution of the orbital elements on a time scale of $10 \mathrm{Myr}$, and also its long-term stability.

\section{Observations}

The speckle interferograms of Gl 22 were recorded on 21 October 2005 with the $6 \mathrm{~m}$ telescope of the Special Astrophysical Observatory (SAO, Russia) with exposure times of $590 \mathrm{~ms}$ each. The detector of the speckle camera was a Rockwell HAWAII array (only one $512 \times 512$ quadrant was used) with a pixel size corresponding to 28.7 mas on the sky. A $K^{\prime}$-band filter with a central wavelength of $2142 \mathrm{~nm}$ and a bandwidth of $293 \mathrm{~nm}$ was used. The direct image is shown in Fig. 1.

The data set for G1 22 consists of 523 speckle interferograms. To compensate the atmospheric speckle transfer function, 568 speckle interferograms of the unresolved star 2MASS $00312573+6738205$ were used. The seeing $(F W H M)$ was $3{ }^{\prime \prime} 0$.

The modulus of the Fourier transform of the object (visibility) was obtained with the speckle interferometry method (Labeyrie 1970). Images with a diffraction-limited resolution of 75 mas were reconstructed from the data set using the bispectrum speckle interferometry method (Weigelt 1977; Weigelt \& Wirnitzer 1983; Lohmann et al. 1983; Hofmann \& Weigelt 1986). The epoch of the observation in fractional Besselian year, the measured separations, position angles, and intensity ratios of the components are listed in Table 1.

\section{The orbits}

The set of available NIR speckle measurements of both pairs has been greatly widened due to observations with the $3.5 \mathrm{~m}$ telescope reported by Woitas et al. (2003), where data on differential photometry in the $K^{\prime}$ band were also given.

\subsection{The actual orbit of the inner pair}

Our first orbit for the Aa-Ab pair was reported by Docobo et al. (2002) and then improved by Docobo \& Tamazian (2006, but also see Docobo et al. 2006). The most recent speckle observations performed with the $6 \mathrm{~m}$ telescope in the $K^{\prime}$ band led to observational residuals of +0.6 and $-0.0^{\prime} 003$ with regard to the improved orbit. It is worth noting that with this measurement, a full revolution has now been completed (see Fig. 2).

\subsection{Is it possible to calculate the outer orbit with a reasonable accuracy?}

Regarding the outer orbit, to date, the secondary covers an arc of $\sim 80^{\circ}$ with respect to $\mathrm{MC}_{A}$. However, we will calculate its most probable preliminary orbital elements according to the methodology that follows.

First, luminosities are obtained on the basis of differential photometry in the $K^{\prime}$ band (Woitas et al. 2003), to which the latest differential photometry data from our Table 1 were added. The empirical masses of each component are then derived on the basis of infrared mass-luminosity relations given by Delfosse et al. (2000) and Henry \& McCarthy (1993) by using weighted means of the above-mentioned differential photometry data. The obtained estimates are given in Table 2.

Furthermore, we obtain the most probable parallax by imposing the following condition: the empirical mass sum of the outer pair $\left(M_{\mathrm{Aa}+\mathrm{Ab}}=0.515 \pm 0.031 M_{\odot}\right)$ must be equal to the dynamical mass determined through a direct application of Kepler's third law. This condition leads to a parallax of $0 . ' 102 \pm 0.001$, which is exactly the same as that obtained by Heintz on the basis of the Sproul plates in 1955. It coincides with the Hipparcos parallax (0.'09874 $\pm 0 . ' 00337)$ within a $1 \sigma$ margin.

\subsection{New determination of the outer orbit}

Although there is a wealth of visual, photographic and CCD measurements of the outer pair, it must be kept in mind that they give the position of $\mathrm{B}$ with respect to the light centre of the inner pair $\mathrm{Aa}-\mathrm{Ab}\left(L C_{A}\right)$, whereas speckle data give the position of $\mathrm{B}$ directly with respect to Aa. Therefore, all measurements must be transformed to positions with respect to $\mathrm{MC}_{A}$.

In our case, this is possible due to the knowledge of 1) the brightness and mass of either component of the inner pair; 2) the precise inner orbit, allowing us to determine the position of each component with respect to both the $\mathrm{MC}_{A}$ and $\mathrm{LC}_{A}$ at any given time. Once these transformations bring the measurements to mutually comparable positions, we can proceed with the orbit calculation by using Docobo's method (Docobo 1985).

Since the parallax is also known, for each generated orbit, we calculate the corresponding total mass. The latter will be a key differentiating factor for selecting the best orbit, since the rms of the $\mathrm{O}-\mathrm{C}$ residuals in the $\theta$ and $\rho$ of generated orbits vary within a narrow margin (see Table 5) and thus do not allow us to choose the orbit with certainty. Therefore, we should search for an orbit with a dynamical mass close to the total mass of the system $0.692 \pm 0.034 M_{\odot}$ given in Table 2 .

With this aim, let us select three base points (listed in Table 4) situated at the beginning, the middle, and the end of the measured arc. As a first approximation, let us give a step of $20^{\circ}$ to the variable $V$ (the difference between the eccentric anomalies corresponding to the base points 3 and 1) appearing in Table 5. It is seen that, indeed, the generated orbits have very similar rms of residuals but that the total mass varies in a relatively wide range. 


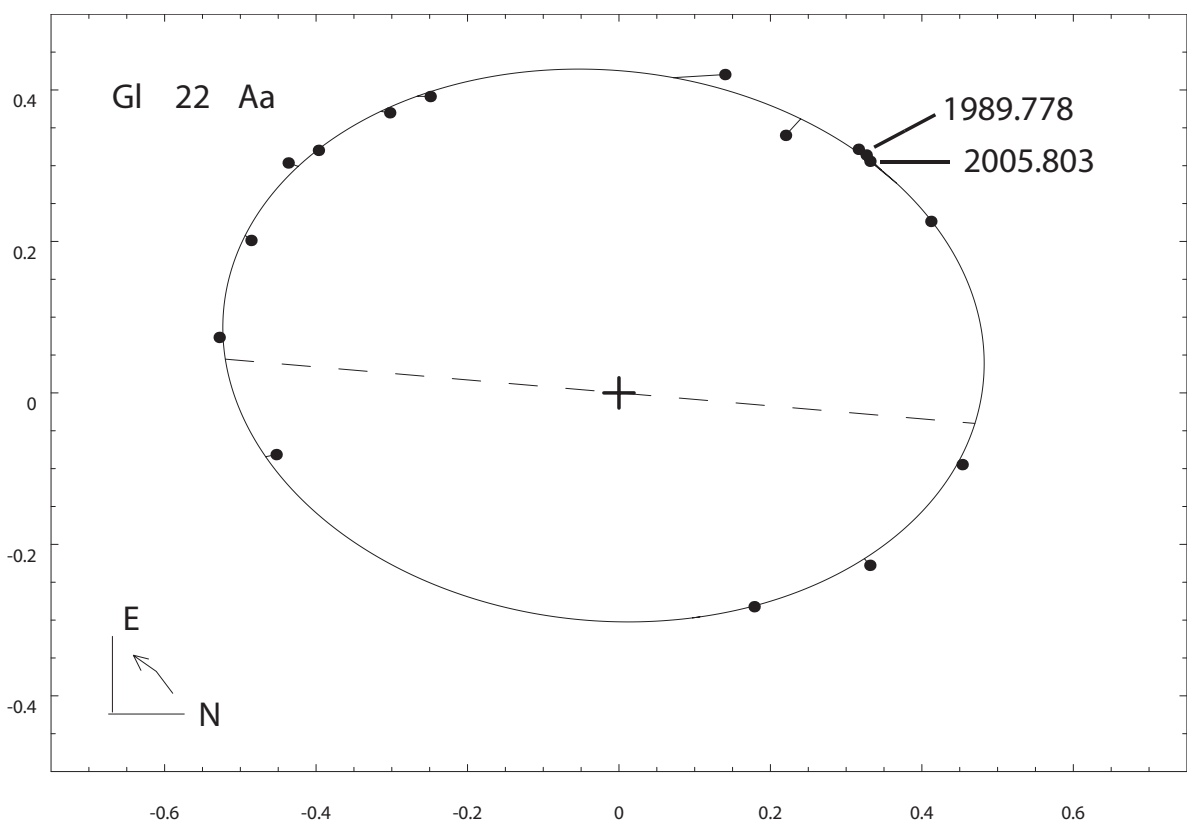

Fig. 2. The apparent orbit of the $\mathrm{Aa}-\mathrm{Ab}$ pair (the scale on both axes is in arcseconds). Each measurement (represented by a dot) is connected to its predicted position by an $\mathrm{O}-\mathrm{C}$ line, and the first and last measurements are marked to show the orbit's closure. The dashed line passing through the primary star is the line of nodes, and the arrow shows the direction of the orbital motion.
Table 2. Luminosity and mass estimates.

\begin{tabular}{|c|c|c|}
\hline 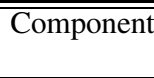 & $\overline{M_{K}}$ & $\begin{array}{l}\text { Mass } \\
\left(M_{\odot}\right)\end{array}$ \\
\hline Aa & 6.42 & $0.377 \pm 0.030$ \\
\hline $\mathrm{Ab}$ & 8.44 & $0.138 \pm 0.007$ \\
\hline B & 7.88 & $0.177 \pm 0.014$ \\
\hline Total mass & & $0.692 \pm 0.034$ \\
\hline
\end{tabular}

Table 3. Orbital elements and system mass.

\begin{tabular}{lcc}
\hline \hline Orbital element & Outer $M C_{A}-B$ pair & Inner Aa- $A b$ pair \\
\hline$P(\mathrm{yr})$ & $223.3_{-9.8}^{+7.2}$ & $15.64 \pm 0.20$ \\
$T$ & $1859.4_{-2.4}^{+5.3}$ & $2000.76 \pm 0.20$ \\
$e$ & $0.293_{-0.025}^{+0.044}$ & $0.174 \pm 0.003$ \\
$a\left({ }^{\prime \prime}\right)$ & $3.322_{-0.060}^{+0.040}$ & $0.511 \pm 0.005$ \\
$i\left(^{\circ}\right)$ & $47.3_{-0.3}^{+0.5}$ & $44.6 \pm 1.5$ \\
$\omega\left(^{\circ}\right)$ & $146.3_{-3.8}^{+2.0}$ & $106.8 \pm 5.0$ \\
$\Omega\left({ }^{\circ}\right)$ & $174.9_{-1.3}^{+2.7}$ & $175.1 \pm 1.0$ \\
\hline
\end{tabular}

Yet, we notice that the masses we are interested in appear in the range $90^{\circ}<V<110^{\circ}$. Therefore, we select this range of $V$ but lower its step to $2^{\circ}$ in the next approximation.

After a second approximation, we find a total mass of $0.69 M_{\odot}$ for $V=105 \mathrm{deg}$, which corresponds to an orbit with a period of $223.4 \mathrm{yr}$. Due to the total mass uncertainty, its values between 0.66 and $0.73 M_{\odot}$ are also valid. Hence, the orbits leading to the masses in this range should be taken into account in order to calculate the standard errors of the orbital elements.

A further improvement of the rms in $\theta$ and $\rho$ leads us to the final orbit with a dynamical mass of $0.693_{-0.062}^{+0.069} M_{\odot}$, shown in Fig. 3 (its elements are listed in Table 3).
Table 4. The base points.

\begin{tabular}{crcl}
\hline \hline No. & $\begin{array}{r}\theta \\
\left({ }^{\circ}\right)\end{array}$ & $\begin{array}{c}\rho \\
\left({ }^{\prime \prime}\right)\end{array}$ & $t$ \\
\hline 1 & 98.05 & 2.603 & 1924.250 \\
2 & 154.24 & 4.000 & 1976.706 \\
3 & 174.28 & 4.010 & 2005.8029 \\
\hline
\end{tabular}

Apart from having a moderate eccentricity (contrary to all previous solutions), the orbit has an inclination and node very similar to those of the inner pair. Assigning indices 1 and 2 to the parameters of the inner and outer orbits, respectively and hereafter in this paper, the mutual inclination can readily be obtained through a well-known expression

$\cos I=\cos i_{1} \cos i_{2}+\sin i_{1} \sin i_{2} \cos \left(\Omega_{2}-\Omega_{1}\right)$

yielding $I=2^{\circ} .7$, which confirms that the orbits, in perfect accordance with Hershey's suggestion, are practically coplanar.

Finally, in Table 8 we present the ephemerides for the position of $\mathrm{B}$ relative to the $\mathrm{MC}_{A}$ and $\mathrm{LC}_{A}$, and its position relative to the main component Aa. Notice that the positions predicted in Table 8 regarding $\mathrm{LC}_{A}$ are useful to test those measurements that cannot resolve the inner pair.

\section{Stability and dynamical evolution of the system}

\subsection{Stability criteria for hierarchical triple systems}

The motion in this hierarchical three-body system is a combination of two perturbed motions: the inner pair $\mathrm{Aa}-\mathrm{Ab}$ and the $\mathrm{B}$ component with regard to $\mathrm{MC}_{A}$.

In order to determine whether or not such a system is stable for a given set of initial orbital elements, several numerical criteria have been proposed by Harrington (1972, hereafter HA), Graziani \& Black (1981, hereafter GB), Eggleton \& Kiseleva (1995, hereafter EK), and Aarseth \& Mardling (1999, hereafter AM).

Both stability and critical parameters are calculated for each of the criteria mentioned above. The system is stable when its stability parameter is larger than the critical one (or smaller for 
Table 5. Approximations.

\begin{tabular}{|c|c|c|c|c|c|c|c|c|c|c|}
\hline $\begin{array}{c}V \\
\left(^{\circ}\right)\end{array}$ & $\begin{array}{c}P \\
(\mathrm{yr})\end{array}$ & $\bar{T}$ & $e$ & $\begin{array}{c}a \\
\left({ }^{\prime \prime}\right)\end{array}$ & $\begin{array}{c}i \\
\left(^{\circ}\right)\end{array}$ & $\begin{array}{c}\Omega \\
\left({ }^{\circ}\right)\end{array}$ & $\begin{array}{c}\omega \\
\left(^{\circ}\right)\end{array}$ & $\mathrm{rms}_{\theta}$ & $\mathrm{rms}_{\rho}$ & $\begin{array}{l}\text { Total } \\
\text { mass } \\
\left(M_{\odot}\right)\end{array}$ \\
\hline \multicolumn{11}{|c|}{ First approximation } \\
\hline 90 & 336.9 & 1928.8 & 0.046 & 4.175 & 52.2 & 162.0 & 292.1 & 0.96 & 0.11 & 0.60 \\
\hline 110 & 198.0 & 1870.7 & 0.411 & 3.181 & 46.9 & 2.1 & 316.4 & 1.32 & 0.15 & 0.77 \\
\hline 130 & 130.7 & 1902.1 & 0.914 & 5.669 & 71.2 & 31.9 & 278.7 & 1.32 & 0.14 & 10.05 \\
\hline \multicolumn{11}{|c|}{ Second approximation } \\
\hline 103 & 234.9 & 1854.77 & 0.249 & 3.387 & 47.8 & 172.2 & 150.0 & 0.96 & 0.11 & 0.66 \\
\hline 104 & 229.0 & 1857.17 & 0.271 & 3.347 & 47.5 & 173.4 & 148.1 & 0.97 & 0.11 & 0.67 \\
\hline 105 & 223.4 & 1859.54 & 0.294 & 3.310 & 47.3 & 174.7 & 146.2 & 0.97 & 0.11 & 0.69 \\
\hline 106 & 217.9 & 1861.86 & 0.317 & 3.277 & 47.1 & 176.0 & 144.3 & 0.97 & 0.11 & 0.70 \\
\hline 107 & 212.7 & 1864.15 & 0.340 & 3.247 & 47.0 & 177.4 & 142.4 & 0.97 & 0.10 & 0.71 \\
\hline
\end{tabular}

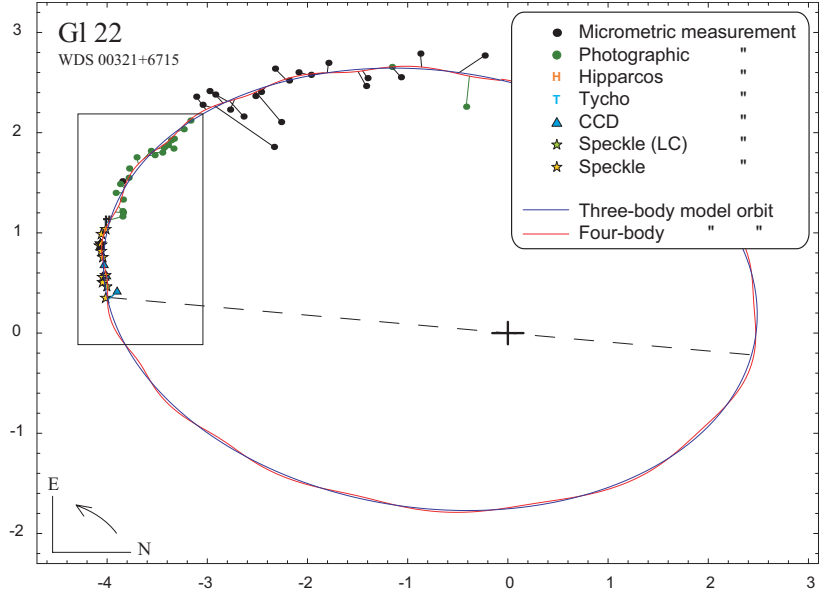

Table 6. Stability margin.

\begin{tabular}{ccc}
\hline \hline$\delta$ & Values & Stability \\
\hline HA & -0.151 & $?$ \\
GB & +0.405 & yes \\
EK & -0.240 & $?$ \\
AM & +0.226 & yes \\
\hline
\end{tabular}

the GB criterion). Otherwise, nothing can be stated regarding its stability.

Thus, the stability margin of a triple system can be defined (Orlov \& Petrova 2000) as

$\delta_{p}= \pm \frac{p-p_{\mathrm{c}}}{p_{\mathrm{c}}}$,

where $p$ represents the stability parameter and $p_{\mathrm{c}}$ the critical parameter ("+" for HA, EK, and AM criteria; "-" for GB criterion).

The larger $\delta_{p}$, the larger the stability margin of the system will be according to the given criterion. Thus, if $\delta_{p}<0$, then the system could be dynamically unstable, i.e. a violation of hierarchy or an escape of the distant component would be possible.

In principle, the small ratio of the (outer to inner) semimajor axis (equal to 6.48) is a sign of the system's instability. With the orbital elements given in Table 3, the stability margin for each criterion is calculated, and results are given in Table 6 .

According to the GB and AM criteria, the system would be stable, whereas those of HA and EK do not allow one to reach the same conclusion. In any case, the obtained values are small enough to clearly decide whether or not the system will be stable. Indeed, it is very close to the stability boundary.

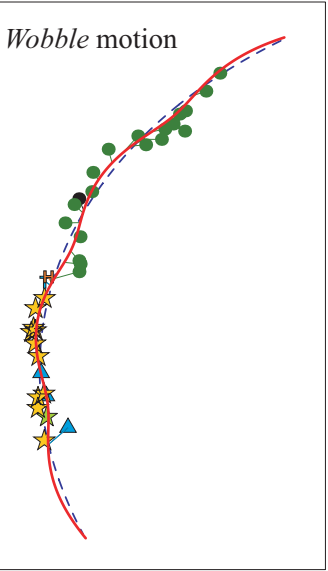

Fig. 3. Left: the apparent orbit of component B relative to the mass centre of $\mathrm{Aa}-\mathrm{Ab}$ (the scale on both axes is in arcseconds). Each measurement (see legend) is connected to its predicted position by an $\mathrm{O}-\mathrm{C}$ line. The dashed line passing through the primary star is the line of nodes, and the arrow shows the direction of the orbital motion. Right: zoom of the orbit showing the wobble motion of the $\mathrm{B}$ component due to the possible new object in the area of measurements with the highest weight.
Table 7. The range of periodic variations of orbital elements at the end of 10 Myr.

\begin{tabular}{lcc}
\hline \hline \multicolumn{1}{c}{ Orbital element } & Outer pair & Inner pair \\
\hline$\Delta P(\mathrm{yr})$ & 4.7 & 0.46 \\
$\Delta T$ & secular & secular \\
$\Delta e$ & 0.023 & 0.095 \\
$\Delta a(\mathrm{AU})$ & 0.457 & 0.097 \\
$\Delta i\left(^{\circ}\right)$ & 1.2 & 4.5 \\
$\Delta \omega\left(^{\circ}\right)$ & secular & secular \\
$\Delta \Omega\left(^{\circ}\right)$ & 1.6 & 6.1 \\
\hline
\end{tabular}

\subsection{Stability and long-term evolution}

To investigate the long-term evolution of the system in a precise manner, a more detailed analysis is necessary. To integrate the hierarchical (nearly-Keplerian) three-body system, we used an implicit Runge-Kutta integrator. All integrations conserve energy and angular momentum to better than $10^{-9}$ and $10^{-4}$, respectively. We explore the orbital elements and parameter space in $10 \mathrm{Myr}$ integrations to demonstrate that neither semimajor axes nor eccentricities exhibit secular variations.

Indeed, as can be seen in Table 7, all orbital elements undergo relatively small periodical variations, except for arguments of periastron that advance secularly ( $\omega_{1}$ and $\omega_{2}$ cover a full revolution in about $10^{4}$ and $5 \times 10^{4}$ yr respectively) and times of periastron passage. Moreover, the mutual inclination remains approximately constant with a maximum periodical variation as small as $\Delta I=0.2$ for the considered interval. 
Table 8. Ephemerides.

\begin{tabular}{lll|ll|ll}
\hline \hline Epoch & \multicolumn{2}{c|}{$B-M C_{A}$ orbit } & \multicolumn{2}{|c|}{$B-L C_{A}$} & \multicolumn{2}{c}{$\mathrm{B}-\mathrm{Aa}$} \\
& $\theta\left({ }^{\circ}\right)$ & $\rho\left({ }^{\prime \prime}\right)$ & $\theta\left(^{\circ}\right)$ & $\rho\left({ }^{\prime \prime}\right)$ & $\theta\left({ }^{\circ}\right)$ & $\rho\left({ }^{\prime \prime}\right)$ \\
\hline 2006.0 & 174.2 & 3.983 & 174.9 & 4.016 & 173.5 & 3.949 \\
2008.0 & 175.5 & 3.991 & 176.3 & 3.982 & 174.7 & 4.001 \\
2010.0 & 177.2 & 3.991 & 177.8 & 3.944 & 176.5 & 4.042 \\
2012.0 & 179.0 & 3.967 & 179.3 & 3.900 & 178.8 & 4.038 \\
2014.0 & 181.1 & 3.905 & 180.8 & 3.852 & 181.4 & 3.961 \\
2016.0 & 183.0 & 3.800 & 182.4 & 3.798 & 183.6 & 3.802 \\
\hline
\end{tabular}

\section{The possible existence of a fourth body}

From a visual inspection of Fig. 3, a weak sinusoidal pattern in the apparent motion of component B has been noticed. This pattern can be attributed to either a very unusual distribution of observational residuals or an unseen fourth body in the system. The latter situation is supported by the fact that such apparent motion has been obtained by considering a collection of different techniques, as can be seen in Fig. 3. In this case, star B would consist of the components $\mathrm{Ba}$ and $\mathrm{Bb}$, whose mass centre will hereafter be defined as $M C_{B}$.

Assuming $\mathrm{Bb}$ to be a very low-mass object of $0.015 M_{\odot}$ $\left(16 M_{J}\right)$ on a circular orbit around $\mathrm{Ba}$ with a period of $\sim 15 \mathrm{yr}$, semimajor axis 0.35 , and coplanar with two other orbits, the observational residuals of the outer orbit are improved. In such a case, the component $\mathrm{Ba}$ would be moving relative to the $\mathrm{MC}_{B}$ on an orbit with a semimajor axis of 0.03 .

The blue line in Fig. 3 corresponds to the motion of $\mathrm{B}$ relative to $\mathrm{MC}_{A}$ (or that of $\mathrm{MC}_{B}$ relative to $\mathrm{MC}_{A}$, if $\mathrm{Bb}$ really does exist), whereas the red line shows the motion of $\mathrm{Ba}$ affected by the putative component Bb. Similar to all visual, photographic, and CCD observations, a single speckle measurement marked as "speckle (LC)" was initially showing the position of B relative to the $\mathrm{LC}_{A}$. For the calculation of orbits, we reduced all such measurements to $\mathrm{MC}_{A}$.

\section{Conclusions}

A methodology for studying the physical and dynamical properties of multiple stars is presented. On this basis, an attempt is made to obtain maximal information on the astrophysical and dynamical properties of the hierarchical triple system of red dwarfs Gl 22.

Recent speckle measurements and differential photometry in the $K^{\prime}$ band allowed us to calculate a better orbital solution for the outer orbit in the hierarchical triple system of the red dwarfs Gl 22 and to confirm that the orbit of the inner pair $\mathrm{Aa}-\mathrm{Ab}$ is almost definitive. The obtained orbital elements, parallax, and individual masses represent a robust system of compatible parameters for this interesting system of low-mass, main-sequence stars exhibiting a low flare activity.

Our results confirm Hershey's suggestion that two orbits are coplanar and co-revolving (Hershey 1973). A newly developed methodology can be applied to the study of triple systems like Gl 22, with an almost definitive inner orbit and a reasonable arc covered by the wide pair.

We have also carried out numerical studies of the stability of the Gl 22 system. The application of four well-known empirical criteria allowed us to conclude that Gl 22 is very close to the boundary of stability. However, the application of an implicit Runge-Kutta integrator extended over 10 Myr showed no significant secular changes in major semi-axes, eccentricities or inclinations.

A weak sinusoidal pattern in the apparent motion of component B has been noticed. If we assume the existence of a very low-mass object "Bb" with a mass of $0.015 M_{\odot}\left(16 M_{J}\right)$ on a circular orbit with a period of $\sim 15 \mathrm{yr}$, the observational residuals of the outer orbit are improved. If confirmed, this would be the first example of a "speckle-astrometric" binary ever discovered. However, a further careful investigation with more extended astrometric and radial velocity data is needed to verify this trend.

Acknowledgements. This paper was financed by research project AYA200407003 and PGIDIT06 PXIB243031PR of Spanish Ministerio de Educación y Ciencia and Xunta de Galicia respectively. This research made use of the Washington Double Star Catalogs maintained at the US Naval Observatory and SIDONIe Database maintained at the Nice-Côte d'Azur Observatory (France).

\section{References}

Aarseth, S. J., \& Mardling, R. A. 1999, in The Dynamics of Small Bodies in the Solar System, a Major Key to Solar System Studies, ed. B. A. Steves, \& A. E. Roy (Dordrecht: Kluwer Academic Publishers), 385

Alden, H. L. 1947, AJ, 52, 138

Delfosse, X., Forveille, T., Ségransan, D., et al. 2000, A\&A, 364, 217

Docobo, J. A. 1985, Celest. Mech., 36, 143

Docobo, J. A., \& Tamazian, V. S. 2006, IAU Comm. 26, 160

Docobo, J. A., Tamazian, V. S., Woitas, J., \& Leinert, Ch. 2002, IAU Comm. 26 Inf. Circ., 147

Docobo, J. A., Tamazian, V. S., Balega, Y. Y., \& Melikian, N. D. 2006, AJ, 132, 994

Eggleton, P., \& Kiseleva, L. 1995, ApJ, 455, 640

Espin, T. E., \& Milburn, W. 1926, MNRAS, 86, 131

Graziani, F., \& Black, D. C. 1981, ApJ, 251, 337

Harrington, R. S. 1972, Celest. Mech., 6, 322

Hartkopf, W. I., \& Mason, B. D. 2007, Sixth Catalog of Orbits of Visual Binary Stars, http://ad.usno.navy.mil/wds/orb6.html

Heintz, W. D. 1955, AN, 282, 221

Heintz, W. D. 1993, PASP, 105, 44

Henry, T. J., \& McCarthy, D. W. 1993, AJ, 106, 773

Hershey, J. L. 1973, AJ, 78, 935

Hofmann, K.-H., Koch, R., \& Weigelt, G. 1986, Mitt. Astron. Gesellschaft, 67, 430

Kirkpatrick, J. D., Henry, T. J., \& McCarthy, D. W., Jr. 1991, ApJS, 77, 417

Labeyrie, A. 1970, A\&A, 6, 85

Lampens, P., \& Strigachev, A. 2001, A\&A, 368, 572

Lohmann, A. W., Weigelt, G., \& Wirnitzer, B. 1983, App. Opt., 22, 4028

McCarthy, Jr., D. W., Henry, T. J., McLeod, B., \& Christou, J. C. 1991, AJ, 101, 214

Orlov, V. V., \& Petrova, A. V. 2000, Astron. Lett., 26, 250

Pettersen, B. R. 1975, A\&A, 41, 113

Söderhjelm, S. 1999, A\&A, 341, 121

Weigelt, G. 1977, Opt. Comm., 21, 59

Weigelt, G., \& Wirnitzer, B. 1983, Opt. Lett., 8, 389

Woitas, J., Tamazian, V. S., Docobo, J. A., \& Leinert, Ch. 2003, A\&A, 406, 293 